\title{
Foot tapping and unilateral vertical jump performance in athletes after knee surgery: an explorative cross-sectional study
}

\author{
Eduard Kurz ${ }^{1 *} \mathbb{0}$, René Schwesig ${ }^{1}$, Stefan Pröger ${ }^{2},{\text { Karl-Stefan Delank }{ }^{1} \text { and Thomas Bartels }}^{2}$
}

\begin{abstract}
Background: Guiding athletes through the rehabilitation process and judging the time at which return to sports can be enabled after anterior cruciate ligament $(\mathrm{ACL})$ injuries are still challenging processes. The purpose of this explorative cross-sectional study was to retrospectively compare unilateral vertical jump as well as vertical foot tapping outcomes in athletes returned to sports after $\mathrm{ACL}$ reconstruction (ACLR) with uninjured athletes.

Methods: Seven-teen ACLR athletes (male/female: 12/5) were examined 11 (6-23) months after their ACL injury and after return to sport clearance together with 67 uninjured athletes (male/female: 51/16). Seventeen age and stature matched controls were selected from the sample of uninjured athletes. Participants unilaterally performed acyclic (squat jump, SJ; drop jump, DJ) and cyclic (foot tapping, FT) tests. SJ peak power, DJ take-off efficiency (TOE) and FT coefficients (FTC) were compared between ACLR and matched as well as unmatched control groups. Limb symmetry index (LSI) as well as performance score were calculated.
\end{abstract}

Results: Analyses of the SJ peak power revealed moderate effects of group (right: $P<0.09, \eta_{p}{ }^{2}=0.06$; left: $P<0.05$, $\eta_{\mathrm{p}}{ }^{2}=0.08$ ). The TOE was largely affected by group (right: $P<0.01, \eta_{\mathrm{p}}{ }^{2}=0.12$; left: $P<0.01, \eta_{\mathrm{p}}{ }^{2}=0.13$ ). No effect of group was found on the FTC $\left(P>0.8, \eta_{\mathrm{p}}{ }^{2}<0.01\right)$. The SJ peak power LSI $(r=0.46, P<0.07)$ and TOE LSI $(r=0.38$, $P=0.13$ ) were positively associated with the performance score of the ACLR group.

Conclusion: Although already returned to sports, the ACLR group underperformed the matched and unmatched control groups significantly. Unilaterally performed vertical jumps may provide additional information on athletes' rehabilitation progress and help to manage the rehabilitation process and decisions on potential readiness after ACLR. More attention should be paid to the direction of the LSI results.

Keywords: Knee joint, Anterior cruciate ligament, Rehabilitation, Return to sports

\section{Background}

Most athletes expect to return to their pre-injury sport without restrictions after a reconstruction of their torn anterior cruciate ligament (ACLR). Different indicators for a safe and successful return to sports have been identified [1]. However, guiding injured patients safely

*Correspondence: eduard.kurz@uk-halle.de

${ }^{1}$ Department of Orthopedic and Trauma Surgery, Martin-LutherUniversity Halle-Wittenberg, Halle (Saale), Germany

Full list of author information is available at the end of the article through the active rehabilitation process and releasing athletes as returned to sports are still challenging processes. Frequently, for different functional outcomes a certain degree of limb symmetry is recommended as a minimal prerequisite. Applying those criteria to a young and athletic population revealed only a low proportion (14\%) of athletes who met all recommended thresholds [2] at the time of return to sports. Moreover, recent results revealed that indicators of absolute performance were superior to limb symmetry when judging athlete's readiness after ACLR [3, 4]. This encourages using both original author(s) and the source, provide a link to the Creative Commons licence, and indicate if changes were made. The images or other third party material in this article are included in the article's Creative Commons licence, unless indicated otherwise in a credit line to the material. If material is not included in the article's Creative Commons licence and your intended use is not permitted by statutory regulation or exceeds the permitted use, you will need to obtain permission directly from the copyright holder. To view a copy of this licence, visit http://creativecommons.org/licenses/by/4.0/. The Creative Commons Public Domain Dedication waiver (http://creativeco mmons.org/publicdomain/zero/1.0/) applies to the data made available in this article, unless otherwise stated in a credit line to the data. 
absolute performance and limb symmetry measures to support the decisions, especially when pre-injury results are not available.

To characterize lower extremity performance, different vertical or horizontal jump or hop tests are routinely used and accepted as a cost-efficient and practicable option to evaluate athletes' leg power capacity [5]. Of those, the squat jump (SJ) and the drop jump (DJ) are considered to provide evidence on athletes' primarily concentric or eccentric dynamic vertical performance ability. The bilateral execution of the SJ and DJ tests are more common, which in turn hampers judgement of unilateral performance. The unilateral alternative can be useful for comparative purposes to judge readiness after a sustained injury. Unilateral SJ was already used to determine functional asymmetries in ACLR participants [6-9].

To address the efficiency of the fast stretch-shortening cycle, various forms of the DJ are frequently applied within strength and conditioning programs. Three different execution strategies are reported in the literature: minimize ground contact time (bounce DJ), maximize jumping height (countermovement DJ), and a mixed strategy in which both demands are considered [10]. From the perspective of talent identification as well as fast stretch-shortening cycle conditioning, the first strategy would probably be the best choice [11]. However, for general conditioning purposes with multiple DJs the second strategy is primarily implemented. The mixed strategy is most commonly applied for performance analyses.

Most recommendations to judge rehabilitation progress or release athletes for a return to sports combine different tests which are acyclic in nature. This aspect was criticized by Maulder and Cronin [12], who encouraged clinicians to use tests examining different qualities of performance. Especially when protocols are applied independently of type of sports or disciplines, a broader range of fundamental performance requirements could add beneficial information. One established cyclic neuromuscular performance test is the vertical foot tapping test [13]. The vertical foot tapping test examines the cyclic speed performance under loaded condition of the lower extremities. Foot tapping tests are considered to be independent of strength qualities and therefore a valuable supplement to strength-related performance tests.

Therefore, the primary purpose of this investigation was to compare performance outcomes of athletes returned to sports after ACLR with uninjured athletes utilizing unilateral vertical jump as well as vertical foot tapping tests. We hypothesized that the ACLR athletes would show performance deficits in the acyclic as well as the cyclic performance tests. The secondary aim was to relate overall performance to symmetry in both groups. We expected a positive association between overall performance and limb symmetry.

\section{Methods \\ Study design and participants}

Eighty-four athletes (handball: 33, soccer: 25, volleyball: 15, ice hockey: 5 , track and field: 3 , judo: 2 , swimming: 1) were included in this cross-sectional study. To be eligible, the following criteria had to be met: no musculoskeletal injury or surgery at the lower extremities in the past 6 months, no vestibular, visual, or hearing impairments. Participants were excluded from the analyzes if they failed to successfully complete any of the required cyclic or acyclic performance tests. The participant characteristics are detailed in Table 1 . Athletes' activity level before injury was assessed using the 11-level Tegner scale

Table 1 Demographic characteristics of the participants presented as means with standard deviations and minimum and maximum

\begin{tabular}{lll}
\hline & ACLR athletes $(\mathbf{n}=\mathbf{1 7})$ & Uninjured athletes $(\mathbf{n}=\mathbf{6 7})$ \\
\hline Male/female & $12 / 5$ & $51 / 16$ \\
Age $($ years $)$ & $25(7,15-39)$ & $23(4,15-35)$ \\
Height $(\mathrm{m})$ & $1.80(0.09,1.65-1.98)$ & $1.84(0.10,1.63-2.06)$ \\
Mass $(\mathrm{kg})$ & $78(15,50-100)$ & $81(12,61-108)$ \\
BMl $\left(\mathrm{kg} / \mathrm{m}^{2}\right)$ & $24.1(3.5,17.4-31.4)$ & $23.8(2.1,19.7-29.0)$ \\
Breakdown of number of athletes from each sport, $\mathrm{n}(\%)$ & & $28(42)$ \\
$\quad$ Handball & $5(29)$ & $17(25)$ \\
$\quad$ Soccer & $8(47)$ & $14(21)$ \\
Volleyball & $1(6)$ & $5(7)$ \\
Ice hockey & $1(6)$ & $2(3)$ \\
Track and field & $2(12)$ & $1(1.5)$ \\
$\quad$ Judo & & \\
Swimming & & \\
\hline
\end{tabular}

$A C L R$ anterior cruciate ligament reconstruction, $B M I$ body mass index 
[14]. The study population comprised 67 uninjured athletes and 17 athletes after completing their rehabilitation phase following their ACLR surgery performed by the same experienced surgeon (TB, hamstring graft) and return to sports clearance $11(6-23)$ months after their ACL injury. Eleven (65\%) ACLR participants had dominant (limb used to kick a ball) side injuries.

Seventeen age and stature matched controls (mCON) were selected from the larger sample of uninjured athletes. Their sides were assigned according to the injured side of their matched peers. The subgroup of mCON $(\mathrm{n}=17)$ was on average 24 (SD 4, 18-31) years old (height: 1.83 (SD 0.11, 1.65-1.97) m, mass: 82 (SD 15, 61-102) kg, BMI: 24.5 (SD 2.5, 19.7-29.0) kg/m²). This investigation was approved by the local ethics committee (approval number: 2016-144) and complied with the Declaration of Helsinki.

\section{Performance tests}

Participants performed acyclic (unilateral vertical jump tests) as well as cyclic (15 s vertical alternating foot tapping (FT) test [15]) tests after a standardized warm-up of 15-min duration (running on a treadmill, a series of bilateral and unilateral jumping drills). Two different types of vertical jumps were performed unilaterally. For the squat jump (SJ), the participant started in a static, semi-squatting position and no preparatory countermovement was allowed. Thus, during SJ a concentric, shortening contraction type (explosive strength) was executed. For the drop jump (DJ), in contrast, the participant had to utilize the fast stretch-shortening cycle (reactive strength). After stepping off a $30 \mathrm{~cm}$ box, the athlete dropped down with the instructions to keep the ground contact time as short as possible and to reach maximum possible jumping height (mixed strategy [10]) and without the heel touching the ground. Especially for the bilaterally executed DJ, the participants are instructed to jump down to a distance of half the individual's body height [16]. Here, the box was located approximately $30 \mathrm{~cm}$ away from the target on the floor to ensure a safe single-leg DJ execution. For each jump test, participants performed 1 familiarization trial followed by 2 attempts used for further evaluation. Each repetition was performed with at least $10 \mathrm{~s}$ rest. The FT test was performed once. To ensure comparability, participants were asked to keep their hands akimbo throughout the entire tests.

\section{Measurement device}

For each test performed, the ground contact times were measured by the contact plates at the center of a SpeedCourt device (GlobalSpeed GmbH, Hemsbach, Germany). This device was implemented for conditioning purposes in healthy and athletic populations [17] as well as in injured athletes aiming to return to pre-injury activities [18]. Flight times (from take-off to landing) obtained by the device were used to calculate jumping heights according to the formulas outlined in Komi and Bosco [19].

\section{Performance outcomes}

Best SJ height was used for further analyses. For the SJ, peak power was additionally estimated from jumping height and body mass using the equation developed and validated by Sayers et al. [20].

To determine best DJ performance, the 2 outcomes 'ground contact time' and 'jumping height' need to be taken into account. Therefore, take-off efficiency (TOE, Eq. 1) was calculated according to Ambarov et al. [21]. This unitless quantity quantifies the speed strength performance, specifically the reactive strength capacity. The DJ attempt with the best TOE was used for further analyses.

$$
T O E=\frac{\text { flight time }(s)^{2}}{\text { ground contact time }(s)}
$$

For the FT test, the ground contact times obtained were averaged separately for each side. The sum of left and right sided contacts were used to determine the FT frequency. Participants' foot tapping performance was quantified as an FT coefficient (FTC, Eq. 2) as introduced by Voss and colleagues [11]. A higher FTC corresponds to a higher cyclic performance capacity.

$$
F T C=\frac{\text { foot tapping frequency }(\mathrm{Hz})}{\text { ground contact time }(\mathrm{ms})} \times 100
$$

Limb symmetry indices (LSI, Barber et al. [22]) were calculated for SJ height, SJ peak power, DJ height, DJ TOE as well as the averaged FT ground contact times. Some athletes' injured sides revealed a better performance, resulting in LSI values greater than 100 percent (cf. [18]). Thus, those results were direction corrected (the smaller value was divided by the larger value [23]) before comparing LSI values statistically.

\section{Overall performance}

The results of the five tests (right and left sided SJ peak power and DJ TOE as well as FTC) were converted into z-scores based on the whole sample of 84 athletes. Z-scores indicate how many standard deviations an individual's score is away from the mean. Accordingly, a $\mathrm{z}$-score of zero equals the sample's mean. Athlete's $\mathrm{z}$-scores were summed-up resulting in the performance score (PS), which indicates overall performance. 


\section{Statistical analysis}

Statistical analyses were carried out with SPSS Statistics 28 (IBM, Armonk, NY, USA) software for Windows. Comparisons of demographic characteristics and symmetry (LSI) between groups were conducted using either independent Student's t tests or Mann-Whitney U tests. To assess subgroup effects on performance outcomes (SJ jumping height, SJ peak power, TOE, FTC) separate univariate analyses of variance were computed. Post-hoc tests on subgroup were performed utilizing the least significant differences test. Statistical significance was set at $P<0.05$. Practical relevance was estimated calculating partial eta squared $\left(\eta_{\mathrm{p}}{ }^{2}\right)$ with values $\geq 0.01, \geq 0.06, \geq 0.14$ indicating small, moderate, or large effects, respectively. Further, linear associations between time since ACL injury and different performance outcomes as well as the overall performance (summed-up z-scores) and LSI values were examined using Pearson's product moment correlation. To unravel possible relationships of the performance outcomes with the level of activity (Tegner scores), the Spearman's rank correlation coefficient was used.

\section{Results}

\section{Participant characteristics}

All participants examined had a median Tegner activity level of seven, which corresponds to a high and competitive activity level. The ACLR athletes did not differ from the selected matched control subgroup concerning their age and their anthropometric characteristics $(P>0.4)$ as well as their Tegner activity level $(U=137.0$, $z=-0.32, P>0.7$, effect size $=-0.06$ ). Two participants (male/female: 1/1) sustained a contact injury, whereas 15 (88\%) participants had a non-contact ACL injury. Fourteen $(82 \%)$ participants performed team sports (soccer: 8, handball: 5 , volleyball: 1 ). Two (12\%) participants have practiced judo and one (6\%) female participant was a javelin thrower.

\section{Unilateral vertical jump performance}

The ACLR limb achieved lower jumping heights compared with their contralateral side as well as both uninjured subgroups. Detailed results on SJ or DJ jumping heights, SJ peak power or DJ take-off efficiency are given in Table 2. The SJ peak power estimations revealed moderate effects of group (injured, matched or right sides: $\left.F(2,81)=4.4, P<0.02, \eta_{\mathrm{p}}{ }^{2}=0.098\right)$. Subsequent post-hoc comparisons indicated differences between the ACLR group and both the matched as well as unmatched control groups $(P<0.02)$ but not between the control subgroups $(P>0.8)$. The take-off efficiency of the DJ was largely affected by group (injured, matched or right sides:
$\left.F(2,81)=11.9, P<0.001, \eta_{\mathrm{p}}^{2}=0.227\right)$. Post-hoc comparisons indicated differences between ACLR and matched as well as unmatched controls $(P<0.001)$, respectively.

\section{Vertical foot tapping performance}

There was no effect of group on the FTC $(F(2,81)=0.1$, $\left.P>0.8, \eta_{\mathrm{p}}{ }^{2}<0.01\right)$. On average, the ACLR athletes (11.6, SD 2.6), the matched (11.4, SD 2.3) and unmatched (11.3, SD 2.2) controls performed equally on the cyclic neuromuscular test. Thus, the FTC results of all participants examined were classified into quartiles (Fig. 1). The results showed that ACLR athletes can be found within each quartile. Furthermore, for ACLR athletes the FTC was positively related to the Tegner activity level $(\rho=0.54, P<0.03)$. Matched controls' Tegner activity level, in contrast, was not associated with the FTC results $(\rho=0.21, P>0.4)$.

\section{Limb symmetry}

For the SJ height, SJ peak power and TOE, 1 ACLR athlete and 9 matched controls showed values greater than 100 . Three ACLR athletes and 10 matched controls revealed values greater than 100 for the DJ height. For the FT contact times, 5 ACLR athletes and 5 matched controls showed values above 100 . The results of the corrected LSI values are displayed in Fig. 2. The direction-corrected LSI values of SJ performance revealed a higher asymmetry in ACLR athletes as compared with the matched controls $(P<0.02$, Mann-Whitney $U$ test $)$. A higher asymmetry between sides in the ACLR group was also found for the DJ outcomes $(P<0.01$, independent Student's t test). No differences could be verified for the direction-corrected LSI values of FT contact times $(P>0.7$, Mann-Whitney U test).

Sixteen (94\%) ACLR athletes revealed at least one LSI value below the $90 \%$ or above the $110 \%$ thresholds. The uncorrected LSI values within the 5 unilaterally examined outcomes are displayed in Fig. 3.

\section{Overall performance}

Overall performance score (PS) was largely affected by group $\left(F(2,81)=5.5, P<0.01, \eta_{\mathrm{p}}{ }^{2}=0.12\right)$. Post-hoc comparisons indicated differences between the ACLR group and the matched $(P=0.009)$ as well as unmatched control group $(P=0.002)$. The direction-corrected SJ peak power LSI $(r=0.46, P<0.07)$ and DJ TOE LSI $(r=0.38$, $P=0.13$ ) was positively associated with the performance score of the ACLR group but not with the performance score of the matched control group $(P>0.4)$.

\section{Inter-relationships}

Time since injury was not associated with either performance outcome $(P>0.1,-0.4<\mathrm{r}<0.4)$. For the ACLR 
Table 2 Participants' jumping heights (cm), squat jump peak power (W) and drop jump take-off efficiency (unitless) presented as means with 95\% confidence intervals

\begin{tabular}{|c|c|c|c|c|}
\hline & \multirow[t]{2}{*}{ ACLR athletes $(n=17)$} & \multicolumn{2}{|c|}{ Uninjured athletes $(n=67)$} & \multirow[t]{2}{*}{$P$ value $^{\mathrm{a}}$} \\
\hline & & $\operatorname{mCON}(n=17)$ & uCON $(n=50)$ & \\
\hline \multicolumn{5}{|l|}{ SJ height } \\
\hline Injured & $13.7(11.1-16.3)$ & & & $<0.001$ \\
\hline Matched & & $20.0(18.1-22.0)$ & & \\
\hline Right & & & $20.4(19.2-21.6)$ & \\
\hline \multicolumn{5}{|l|}{ SJ height } \\
\hline Uninjured & $17.3(14.9-19.8)$ & & & 0.03 \\
\hline Matched & & $20.6(18.4-22.8)$ & & \\
\hline Left & & & $21.0(19.6-22.4)$ & \\
\hline \multicolumn{5}{|l|}{ DJ height } \\
\hline Injured & $13.1(10.7-15.4)$ & & & $<0.001$ \\
\hline Matched & & $17.5(15.9-19.2)$ & & \\
\hline Right & & & $17.9(16.6-19.2)$ & \\
\hline \multicolumn{5}{|l|}{ DJ height } \\
\hline Uninjured & $17.3(15.8-18.8)$ & & & 0.48 \\
\hline Matched & & $17.6(15.7-19.4)$ & & \\
\hline Left & & & $18.4(17.3-19.6)$ & \\
\hline \multicolumn{5}{|l|}{ SJ peak power } \\
\hline Injured & 2320 (1906-2735) & & & $<0.02$ \\
\hline Matched & & 2882 (2499-3264) & & \\
\hline Right & & & $2842(2679-3005)$ & \\
\hline \multicolumn{5}{|l|}{ SJ peak power } \\
\hline Uninjured & 2545 (2146-2944) & & & 0.18 \\
\hline Matched & & 2914 (2515-3313) & & \\
\hline Left & & & 2875 (2708-3042) & \\
\hline \multicolumn{5}{|c|}{ DJ take-off efficiency } \\
\hline Injured & $0.35(0.28-0.43)$ & & & $<0.001$ \\
\hline Matched & & $0.59(0.52-0.66)$ & & \\
\hline Right & & & $0.59(0.53-0.64)$ & \\
\hline \multicolumn{5}{|c|}{ DJ take-off efficiency } \\
\hline Uninjured & $0.50(0.45-0.55)$ & & & 0.16 \\
\hline Matched & & $0.57(0.50-0.64)$ & & \\
\hline Left & & & $0.59(0.54-0.64)$ & \\
\hline
\end{tabular}

$A C L R$ anterior cruciate ligament reconstruction, $D J$ drop jump, $m C O N$ matched controls, $u C O N$ unmatched controls, $S J$ squat jump

${ }^{a} P$ value of univariate analysis of variance

group, the FTC was positively associated with the SJ height $(r=0.50, P<0.05)$, SJ peak power $(r=0.50, P<0.04)$, DJ height $(r=0.65, P<0.01)$ and TOE $(r=0.68, P<0.01$, Fig. 4$)$ at the uninjured side but not with the results at the injured side $(P>0.06)$. Interestingly, no such relationships could be verified for the matched control group at either side.

\section{Discussion}

This study investigated lower extremity functional performance of acyclic as well as cyclic demands in ACLR athletes who were already released for return to sports.
Overall performance as well as limb symmetry was lower in ACLR athletes. Cyclic performance as depicted by the $15 \mathrm{~s}$ vertical foot tapping test was comparable between groups. Unilateral vertical jump performances were markedly lower in the ACLR group. Symmetry of the unilateral vertical jumps correlated at least weakly with overall performance in ACLR athletes only.

\section{Unilateral vertical jump performance}

To quantify deficits of specific strength qualities, squat, countermovement and drop jumps are most commonly 


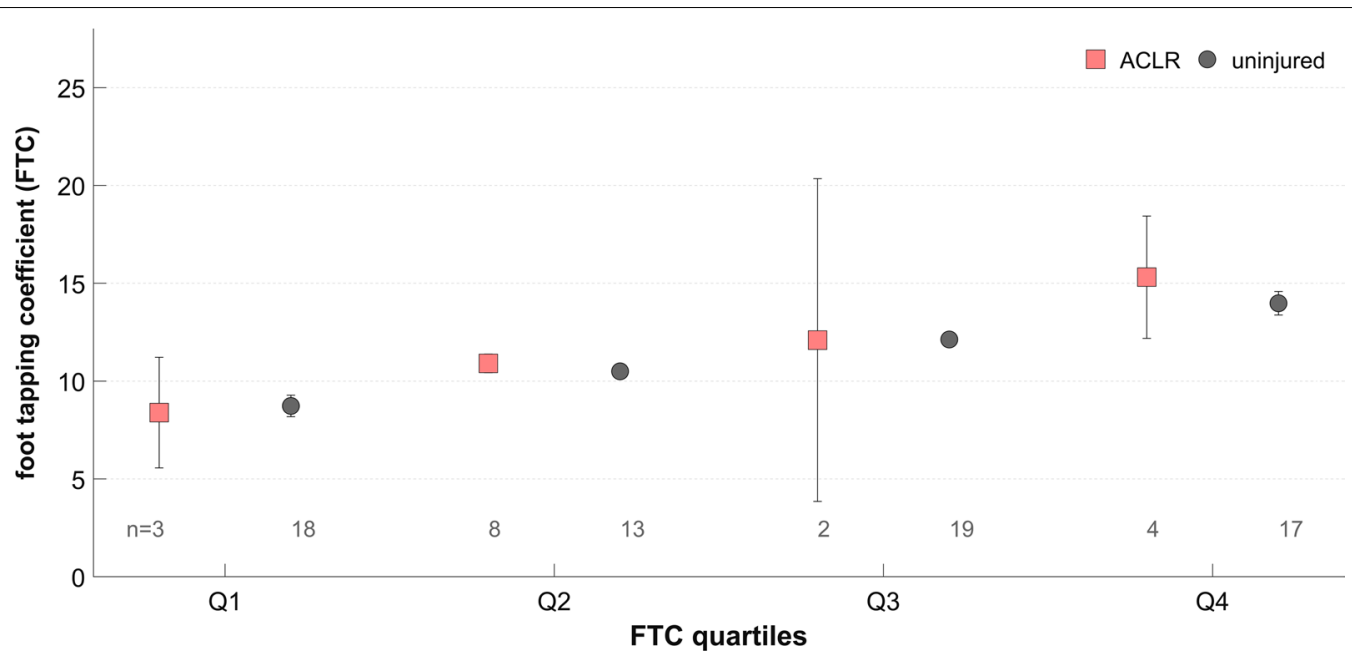

Fig. 1 Results of foot tapping coefficients (FTC) of ACLR $(n=17)$ and uninjured $(n=67)$ athletes separated into quartiles. Values presented as mean with $95 \%$ confidence intervals

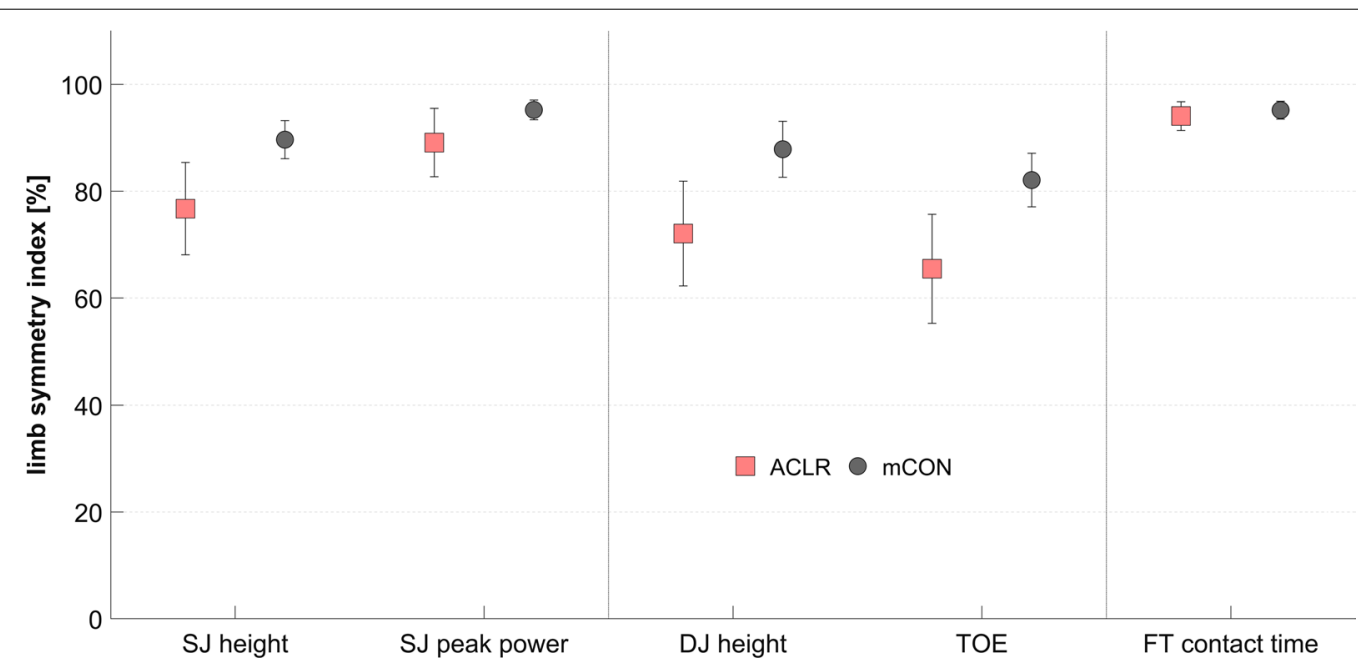

Fig. 2 Direction-corrected limb symmetry indices of ACLR athletes $(n=17)$ and the matched controls ( $m C O N, n=17)$ of squat jump (SJ, jumping height and peak power), drop jump (DJ, jumping height and take-off efficiency, TOE) and foot tapping (FT) contact time outcomes. Values presented as mean with 95\% confidence intervals

applied in a bilateral fashion. In addition to generally used horizontal hop tests in ACLR subjects, Hopper and colleagues [6] employed a vertical jump unilaterally to enable comparisons between sides. Their flight times recorded on a force plate from reconstructed (0.21$0.44 \mathrm{~s})$ and uninjured (0.31-0.47 s) legs are comparable to those recorded with our device (injured: $0.20-0.43 \mathrm{~s}$, uninjured: $0.23-0.45 \mathrm{~s}$ ). Pairot de Fontenay et al. [8] examined a comparable sample of ACLR athletes (11 males) cleared for return to sports. They found a $24 \%$ lower jumping height at the injured side. Our male athletes revealed a $20 \%$ lower jumping height on average at the injured side. However, as can be seen in Fig. 3, one athlete reached a $21 \%$ higher jumping height on his injured side. This finding underpins the direction specificity of the LSI values, which the clinician must keep in mind. The 'direction of asymmetry' was emphasized by Impellizzeri et al. [24] and Maloney [25]. From a practical perspective it may not be irrelevant which limb performed better or worse.

Since reactive strength qualities are basic requirements for jumping, hopping, or running activities, injured athletes need to address the reactive capacity throughout the rehabilitation process. The DJ outcomes revealed the 

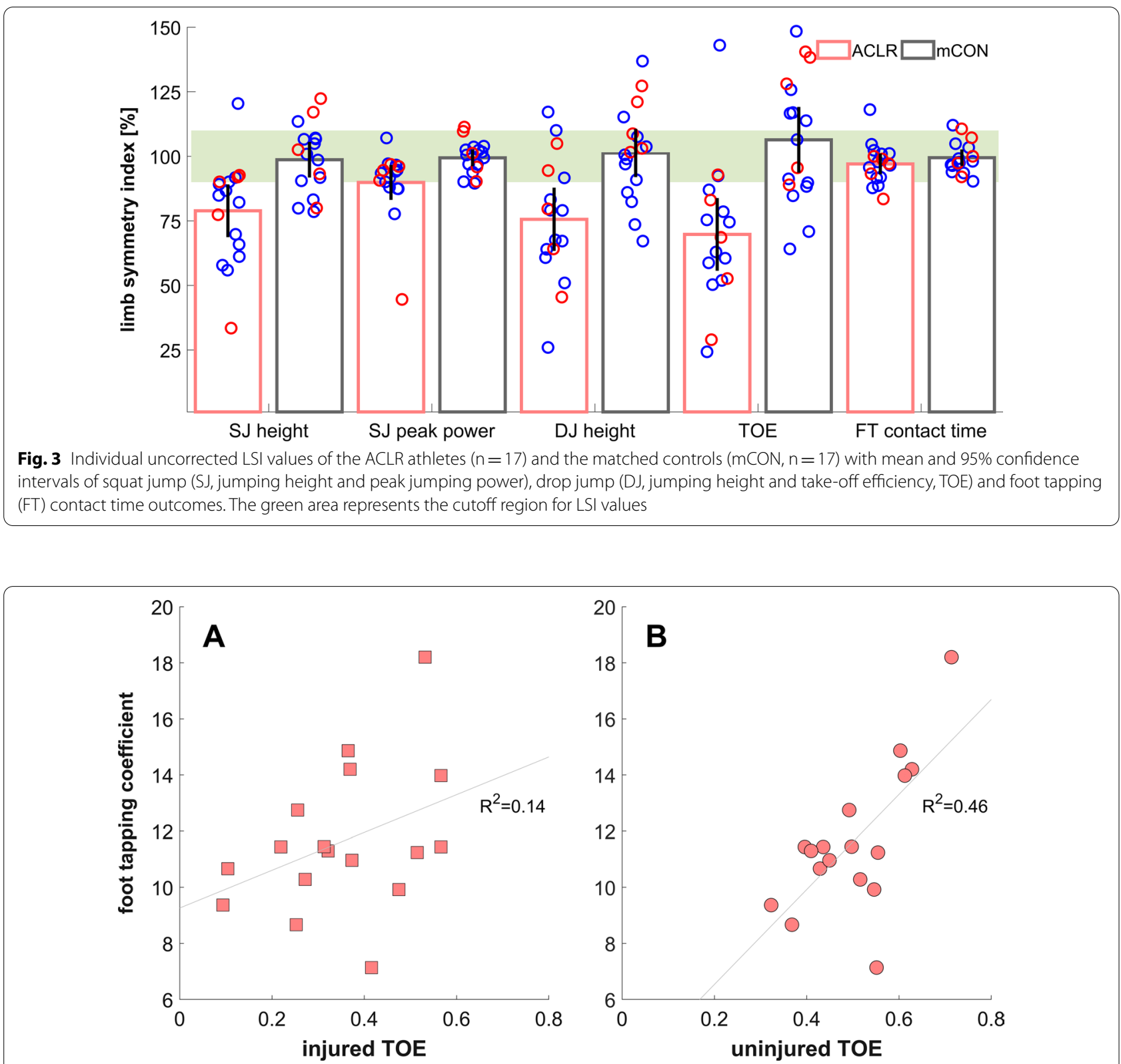

Fig. 4 Association between the drop jump take-off efficiency (TOE) and the foot tapping coefficients at the injured (A) and uninjured (B) sides of the ACLR athletes $(n=17)$

largest differences between subgroups. Single-leg reactive neuromuscular performance was affected most by the ACL injury. After nine months post surgery, King et al. [26] found smaller side effects in front hop performance as compared with unilateral DJ. They concluded that at that rehabilitation stage the horizontal-type hop test may overestimate the actual functional status. In contrast to the front hop, the DJ executed with an immediate vertical take-off utilizes the fast stretch-shortening cycle. The jumping strategy used to absorb the impact forces during
DJ execution results in shorter or longer ground contact times. With shorter ground contact times, the leg and ankle stiffness will increase [27] and therewith the TOE. Despite the fact that lower extremity stiffness is associated with athletic performance, higher peak forces bear risks for cartilage overload or bony injuries [28]. Investigating DJ kinematics, uninjured female athletes showed different landing patterns [29]. Stiff, knee or hip dominant landing strategies were distinguished according to degrees of joint flexion demonstrated. Lower hip and 
knee joint flexion landing angles (greater dominance) were found for bilateral versus unilateral DJ executions. This may indicate more effective force absorption in double-legged DJ. Consequently, after a sustained ACL injury, one useful strategy might be to realize larger joint moments [30] to compensate for vertical impacts at the expense of stretch-shortening cycle efficiency. This leads to longer ground contact times and limited TOE. Therefore, TOE can be interpreted as a surrogate for lower extremity stiffness.

\section{Vertical foot tapping performance}

Tapping tests are routinely applied in clinical environments to assess neurological dysfunction in upper and lower extremities [31]. In professional sports, tapping tests examine cyclic neuromuscular performance and are used for talent identification [11]. Although lower extremities' cyclic performance was on average similar between injured and uninjured athletes, the results of the ACLR participants corresponded to their Tegner activity level. However, due to the relatively long duration of the FT test $(15 \mathrm{~s})$, the individual's anaerobic capacity very likely influenced the results [15]. In ACLR athletes, each of the unilateral vertical jump performance outcomes was moderately correlated with the FTC, but only at the uninjured side. This suggests that the ACL injury affects the person as a whole, and different qualities of performance are less independent from each other in injured athletes.

\section{Limb symmetry and overall performance}

Only the acyclic performance outcomes revealed substantial side-to-side differences, which were larger for the ACLR athletes. Interestingly, for TOE only 1/17 ACLR athletes and 4/17 matched controls reached the threshold value of $90 \%$. Some concerns exist surrounding the utilization of LSI values as the only discharge criteria. Wellsandt et al. [3] followed 70 ACLR patients, eleven of whom sustained a second injury, and found that the estimated pre-injury capacity was more sensitive in predicting a second injury than the LSI values. In another study, 64 ACLR patients were followed up to 2 years after completing the rehabilitation program [4]. Although all patients finished the program with LSI values $>90 \%$, at the end of rehabilitation only 46 successfully returned to their pre-injury level. Compared to those patients who failed to return, they showed higher absolute scores on all hop tests performed.

\section{Limitations and future directions}

The participants in this study were recruited from a rehabilitation center and completed routine performance measures, best representing a real-life cohort.
Consequently, this study is limited to a relatively small and heterogeneous sample. Since acute fatigue was shown to alter intrinsic risk factors in non-contact ACL injured participants [32], future studies should elaborate effects of different protocols as well as task specificity. In this context, in particular the execution strategies of the drop jumps should be considered more consistently in assessments of athletes' readiness. Besides quantitative evaluations, movement quality ratings (e.g., drop jump landing) would complement the competency profile.

\section{Conclusion}

Based on the tests utilized in this study, the ACLR group underperformed the matched and unmatched control groups significantly, although already returned to sports. Unilaterally performed vertical jumps may provide additional information on athletes' rehabilitation progress and therefore help to manage the rehabilitation process and decisions on potential readiness after ACLR. More attention should be paid to the direction of the LSI results.

\section{Abbreviations}

ACL: Anterior cruciate ligament; ACLR: Anterior cruciate ligament reconstruction; DJ: Drop jump; $\eta_{p}^{2}$. Partial eta squared; FT: Foot tapping; FTC: Foot tapping coefficient; LSI: Limb symmetry index; mCON: Matched controls; PS: Performance score; SJ: Squat jump; TOE: Take-off efficiency; uCON: Unmatched controls.

\section{Acknowledgements}

The investigators highly appreciate the athletes' compliance and trust. The authors would like to thank Ms. Tara Russell for language assistance.

\section{Authors' contributions}

EK, RS, SP, KSD and TB contributed to the original idea, study design and protocol, the conception of the work, conducting the study, revising the drafting, and editing of the article. SP collected data. EK and SP contributed to data analysis. EK drafted the first version of the manuscript. All authors approved the final version of the article. All authors read and approved the final manuscript.

\section{Funding \\ Not applicable.}

\section{Availability of data and materials}

The datasets used and analyzed during the current study are available from the corresponding author on reasonable request.

\section{Declarations}

Ethics approval and consent to participate

The Institutional Review Board of Martin-Luther-University Halle-Wittenberg approved the study protocol (approval number: 2016-144). The participants were informed about the details of the study and provided written informed consent before study enrolment. Informed consent was obtained from all the participants, and procedures were conducted according to the Declaration of Helsinki.

Consent for publication

Participants consent for publication was not required. 


\section{Competing interests}

The authors declare no competing interests with respect to the research, authorship, and/or publication of this article.

\section{Author details}

${ }^{1}$ Department of Orthopedic and Trauma Surgery, Martin-Luther-University Halle-Wittenberg, Halle (Saale), Germany. ${ }^{2}$ Joint Surgery Center, Sports Clinic Halle, Halle (Saale), Germany.

Received: 20 October 2021 Accepted: 14 February 2022

Published online: 03 March 2022

\section{References}

1. Kyritsis P, Bahr R, Landreau P, Miladi R, Witvrouw E. Likelihood of ACL graft rupture: not meeting six clinical discharge criteria before return to sport is associated with a four times greater risk of rupture. Br J Sports Med. 2016;50(15):946-51.

2. Toole AR, Ithurburn MP, Rauh MJ, Hewett TE, Paterno MV, Schmitt LC. Young athletes cleared for sports participation after anterior cruciate ligament reconstruction: how many actually meet recommended returnto-sport criterion cutoffs? J Orthop Sports Phys Ther. 2017;47(11):825-33.

3. Wellsandt E, Failla MJ, Snyder-Mackler L. Limb symmetry indexes can overestimate knee function after anterior cruciate ligament injury. J Orthop Sports Phys Ther. 2017;47(5):334-8.

4. Welling W, Benjaminse A, Lemmink K, Gokeler A. Patient reported outcome measures and return to sport rates 2 years after anterior cruciate ligament reconstruction. In: Roi GS, Della Villa S, editors. Football medicine meets the universe of sport: proceedings of the XXVIII isokinetic medical group international conference. Torgiano: Calzetti \& Mariucci Editori; 2019. p. 37-8.

5. Keir PJ, Jamnik VK, Gledhill N. Technical-methodological report: a nomogram for peak leg power output in the vertical jump. J Strength Cond Res. 2003;17(4):701-3.

6. Hopper DM, Goh SC, Wentworth LA, Chan DYK, Chau JHW, Wootton GJ, et al. Test-retest reliability of knee rating scales and functional hop tests 1 year following anterior cruciate ligament reconstruction. Phys Ther Sport. 2002:3(1):10-8.

7. Katayama M, Higuchi H, Kimura M, Kobayashi A, Hatayama K, Terauchi M, et al. Proprioception and performance after anterior cruciate ligament rupture. Int Orthop. 2004;28(5):278-81.

8. Pairot de Fontenay B, Argaud S, Blache Y, Monteil K. Motion alterations after anterior cruciate ligament reconstruction: comparison of the injured and uninjured lower limbs during a single-legged jump. J Athl Train. 2014:49(3):311-6.

9. Lee DW, Yang SJ, Cho SI, Lee JH, Kim JG. Single-leg vertical jump test as a functional test after anterior cruciate ligament reconstruction. Knee. 2018;25(6):1016-26.

10. Young WB, Pryor JF, Wilson GJ. Effect of instructions on characteristics of countermovement and drop jump performance. J Strength Cond Res. 1995:9(4):232-6.

11. Voss G, Witt M, Werthner R. Herausforderung Schnelligkeitstraining. Aachen: Meyer \& Meyer; 2007. p. 223.

12. Maulder P, Cronin J. Horizontal and vertical jump assessment: reliability, symmetry, discriminative and predictive ability. Phys Ther Sport. 2005:6:74-82.

13. Zallinger G, Müller E. Charakteristik der Beintapping-Bewegung unter dem Aspekt ausgewaehlter EMG-Parameter. Spectrum der Sportwissenschaften. 1999;11(Suppl.):79-86.

14. Tegner Y, Lysholm J. Rating systems in the evaluation of knee ligament injuries. Clin Orthop Relat Res. 1985;198:43-9.

15. Krauss TT. Der 15 Sekunden Foot-Tapping Test (FTT15): Evaluation als sportmotorisches Testverfahren sowie Analyse der Beeinflussbarkeit leistungsphysiologischer Parameter durch eine spezifische Vorbelastung. Hamburg: Medizinische Fakultät der Universität Hamburg; 2011. p. 129.

16. Padua DA, Marshall SW, Boling MC, Thigpen CA, Garrett WE Jr, Beutler Al. The Landing Error Scoring System (LESS) is a valid and reliable clinical assessment tool of jump-landing biomechanics: the JUMP-ACL study. Am J Sports Med. 2009;37(10):1996-2002.
17. Düking P, Born DP, Sperlich B. The SpeedCourt: reliability, usefulness, and validity of a new method to determine change-of-direction speed. Int J Sports Physiol Perform. 2016;11(1):130-4.

18. Achenbach L, Krutsch W, Koch M, Zeman F, Nerlich M, Angele P. Contact times of change-of-direction manoeuvres are influenced by age and the type of sports: a novel protocol using the SpeedCourt system. Knee Surg Sports Traumatol Arthrosc. 2019;27(3):991-9.

19. Komi PV, Bosco C. Utilization of stored elastic energy in leg extensor muscles by men and women. Med Sci Sports. 1978;10(4):261-5.

20. Sayers SP, Harackiewicz DV, Harman EA, Frykman PN, Rosenstein MT. Cross-validation of three jump power equations. Med Sci Sports Exerc. 1999;31(4):572-7.

21. Ambarov E, Kuporosov B, Matveev V. Trenirovka bystroty ottalkivanija (Training der Absprungschnelligkeit). Legkaja atletika. 1982;9:20.

22. Barber SD, Noyes FR, Mangine RE, McCloskey JW, Hartman W. Quantitative assessment of functional limitations in normal and anterior cruciate ligament-deficient knees. Clin Orthop Relat Res. 1990;255:204-14.

23. Daniel D, Malcolm L, Stone ML, Perth H, Morgan J, Riehl B. Quantification of knee stability and function. Contemp Orthop. 1982;5(1):83-91.

24. Impellizzeri FM, Rampinini E, Maffiuletti N, Marcora SM. A vertical jump force test for assessing bilateral strength asymmetry in athletes. Med Sci Sports Exerc. 2007;39(11):2044-50.

25. Maloney SJ. The relationship between asymmetry and athletic performance: a critical review. J Strength Cond Res. 2019;33(9):2579-93.

26. King E, Richter C, Franklyn-Miller A, Daniels K, Wadey R, Moran R, et al. Whole-body biomechanical differences between limbs exist 9 months after ACL reconstruction across jump/landing tasks. Scand J Med Sci Sports. 2018;28(12):2567-78.

27. Arampatzis A, Schade F, Walsh M, Brüggemann GP. Influence of leg stiffness and its effect on myodynamic jumping performance. J Electromyogr Kinesiol. 2001:11(5):355-64.

28. Brazier J, Maloney S, Bishop C, Read PJ, Turner AN. Lower extremity stiffness: considerations for testing, performance enhancement, and injury risk. J Strength Cond Res. 2019;33(4):1156-66.

29. Dingenen B, Malfait B, Vanrenterghem J, Robinson MA, Verschueren SMP, Staes FF. Can two-dimensional measured peak sagittal plane excursions during drop vertical jumps help identify three-dimensional measured joint moments? Knee. 2015:22(2):73-9.

30. Fox AS. Change-of-direction biomechanics: Is what's best for anterior cruciate ligament injury prevention also best for performance? Sports Med. 2018;48(8):1799-807.

31. Gunzler SA, Pavel M, Koudelka C, Carlson NE, Nutt JG. Foot-tapping rate as an objective outcome measure for Parkinson disease clinical trials. Clin Neuropharmacol. 2009;32(2):97-102.

32. Verschueren J, Tassignon B, De Pauw K, Proost M, Teugels A, Van Cutsem J, et al. Does acute fatigue negatively affect intrinsic risk factors of the lower extremity injury risk profile? A systematic and critical review. Sports Med. 2020;50(4):767-84.

\section{Publisher's Note}

Springer Nature remains neutral with regard to jurisdictional claims in published maps and institutional affiliations.

Ready to submit your research? Choose BMC and benefit from:

- fast, convenient online submission

- thorough peer review by experienced researchers in your field

- rapid publication on acceptance

- support for research data, including large and complex data types

- gold Open Access which fosters wider collaboration and increased citations

- maximum visibility for your research: over 100M website views per year

At BMC, research is always in progress.

Learn more biomedcentral.com/submissions 